Artículo de Revisión

\section{Enfrentamiento Práctico del Paciente con Síndrome de Boca Urente, una Propuesta para el Estudio y Tratamiento}

\author{
Rubén González ${ }^{1}$, Gonzalo Rojas²
}

\begin{abstract}
RESUMEN
El Síndrome de Boca Urente (SBU) clásicamente se define como un dolor oral quemante sin hallazgos en el examen físico o de laboratorio. Muchas veces puede ser asociado a procedimientos dentales o alteraciones psiquiátricas pero en la gran mayoría de los casos, no existe un fenómeno relacionado. Sin embargo, no existe consenso en su evaluación clínica, lo que se refleja en lo heterogéneo de los estudios de prevalencia, patogenia y tratamiento. Es por esto que muchas veces, representa un desafio el diagnóstico y tratamiento, especialmente cuando especialmente cuando existe un serio deterioro de la calidad de vida, sin una causa identificable. Este artículo tiene como objetivo contribuir en la identificación de causas y en la definición de pautas para el diagnostico y manejo clínico del SBU, proponiendo un esquema de estudio y manejo en base a la revisión de la literatura y la experiencia clínica.
\end{abstract}

Palabras clave: Sindrome de Boca Urente; Dolor, Enfermedad Oral; Dermatologia

\section{SUMMARY}

Classically, we can define Burning-Mouth Syndrome (BMS) as burnish oral pain with no physical or laboratory findings; often can be associated with dental procedures or psychiatric disorders but in general, there is no related phenomenon. Even today there is confusion and ignorance of this syndrome, being reflected in the heterogeneity of the studies of prevalence, pathogenesis and treatment. This is why, in clinical practice, study and treatment are challenging, especially when we are faced with a patient who feels seriously impaired in his quality of life and does not find the cause. This article attempts to contribute to the understanding of the BMS, proposing a study and management scheme based on literature review and clinical experience.

Key words: Burning Mouth Syndrome; Pain; Mouth Diseases; Dermatology
$\mathrm{L}$ a Asociación Internacional para el estudio del dolor (IASP) define el síndrome de boca urente (SBU) como un dolor quemante en la lengua u otra estructura de la mucosa oral persistente por al menos cuatro meses, asociados a aparente indemnidad de la mucosa oral y exámenes de laboratorio normales. ${ }^{1}$ Los criterios diagnósticos de la IASP son los siguientes: sensación de ardor en la lengua u otras partes de la mucosa oral, usualmente bilateral y asociada con disgeusia, intolerancia a la boca seca y a la prótesis dental. A su vez, la Sociedad Internacional de Cefaleas (IHS) describe el SBU como una sensación urente intraoral, donde no puede ser encontrada una causa médica o dental, persistente por al menos 3 meses. ${ }^{2}$ El IHS, además, señala que el dolor puede limitarse a la lengua (glosodinia), con síntomas asociados que incluyen: sequedad subjetiva de la boca (xerostomía), parestesia y alteración del gusto. Según sus características fisiopatológicas podemos clasificar el SBU en primario, si no hay causa conocida; o secundario, en la cual podemos identificar un factor relacionado, siendo la disminución de la producción de saliva (hiposialia) una causa importante, que a su vez, puede ser secundaria a patologías como el Síndrome Sjögren y otras enfermedades reumatológicas, o por factores externos como radiotratamiento o efecto adverso a fármacos. ${ }^{3}$
${ }^{1}$ Departamento

Dermatología,

Facultad de

Medicina,

Universidad de

Chile. Santiago.

Chile.

${ }^{2}$ Departamento de

Patología y Medicina

Oral, Facultad

de Odontología,

Universidad de

Chile. Santiago,

Chile.

Trabajo no recibió financiamiento.

Los autores declaran

no tener conflictos

de interés.

Recibido el 1 de agosto de 2018 ,

aceptado el 21 de

febrero de 2019.

Correspondencia a:

Rubén González

Guevas

E-Mail: ruben. gonzalez.c@gmail.

com

Dirección: Santa

Victoria 360,

Departamento

1604, Santiago

Centro, Región

Metropolitana,

Chile. Código

postal 8330330. 


\section{EPIDEMIOLOGÍA}

Se estima que la prevalencia del SBU es de entre un $0.7 \%$ y un $4,6 \%$ de la población general. Esta gran variabilidad se explicaría por la falta de criterios diagnósticos estrictos, y diferencias en la metodología usada para su diagnóstico (encuesta o evaluación clínica), no estableciéndose aún una prevalencia fidedigna en la población general.

El SBU raramente se presenta antes de los treinta años y no ha sido descrito en niños ni adolescentes. Además, suelen referir algún tipo trastorno psicológico asociado. $^{4}$

Es más frecuentemente reportado por mujeres en la quinta a séptima década de vida, presentándose entre tres y doce años después del inicio de la menopausia, con una proporción entre mujeres y hombres que varía de 3:1 a 16:1. Sin embargo, un estudio realizado en Japón, no logró establecer una diferencia estadísticamente significativa entre hombres y mujeres, mostrando una prevalencia del $2,8 \%$ para hombres y $3,2 \%$ para mujeres. ${ }^{5,6,7}$

\section{ETIOPATOGENIA}

Se ha relacionado el SBU con factores de tipo local, sistémico y psicológico. Esto demuestra que es un síndrome complejo en el que podemos reconocer diferentes subtipos. En el SBU llamado primario, esencial o idiopático, no se pueden identificar clínicamente alteraciones orgánicas, locales o sistémicas, a las cuales atribuir del trastorno; por el contrario, en el SBU secundario, es posible atribuir las molestias a lesiones observables, o a causas conocidas, y que, al tratarlas, mejoran el cuadro clínico.

Si bien en el SBU primario no se ha podido encontrar una causa directa, en los últimos años numerosos estudios han reportado indicios de un mecanismo neuropático en su génesis, aunque existen controversias sobre si este mecanismo es periférico o central. Por ejemplo, Grushka reportó en pacientes con SBU, una menor tolerancia térmica en punta de la lengua, sin embargo, esta elevación del umbral en la percepción térmica, parece también estar relacionada con un componente afectivo emocional. Así mismo, Jääskeläinen et al. observaron que con la excitación-inhibición del nervio trigémino se producía una alteración tanto en la percepción del dolor como en la habituación al estímulo en los pacientes con SBU. ${ }^{8}$

Además de estas observaciones perceptivas, se han evidenciado alteraciones en la mucosa anterolateral de la lengua, caracterizadas por una baja densidad de fibras finas sensitivas subepiteliales, lo que se ha relacionado con una degeneración axonal a este nivel. ${ }^{9,10}$ También se ha obtenido evidencias de un posible mecanismo neuropático, debido a una posible hiperactividad del sistema motor y sensorial del nervio trigémino, seguida de una inhibición central, como resultado del daño gustativo en el nervio de la cuerda del tímpano o del glosofaríngeo, secundario a un desorden temporomandibular. ${ }^{11}$ También se ha observado una percepción somatosensorial y degustativa disminuida en la lengua. A través de la resonancia magnética funcional del talamo, se ha demostrado un patrón anormal tras la estimulación dolorosa y térmica, con una disminución de captación en el putamen de los pacientes con SBU, por lo que también se postula una posible alteración del sistema dopaminérgico nigroestriado. ${ }^{12}$

\section{EsTUDIO}

El abordaje del SBU requiere de estudio para descartar posibles causas secundarias, las que pueden ser locales, sistémicas o psicopatológicas, y que debiesen ser tratadas para la resolución del cuadro, y en caso no encontrarlas, realizar el manejo adecuado de un SBU primario. Para tales propósitos sugerimos considerar los siguientes puntos (figura 1):

Historia clínica: realizar una anamnesis detallada es imprescindible para identificar antecedentes médicos, personales y familiares, cuantificando la sensación dolorosa y describiendo la calidad, duración y cronología de los síntomas. Se debe prestar especial atención a la ingesta de fármacos que pudiesen producir hiposialia (Tabla 1) así como presencia de hábitos parafuncionales, que son aquellos del sistema masticatorio que no tienen sentido útil, que son involuntarios e incluso desconocidos para el paciente, como el bruxismo, morder los labios, mejillas, lengua entre otros. Es importante un interrogatorio psiquiátrico para descartar una patología del ánimo subyacente, siendo recomendable la evaluación por psiquiatría, ayudando a tener un enfoque distinto, aportando tanto para el diagnóstico como en tratamiento del SBU. ${ }^{13,14,15}$ 
Tabla 1

Drogas asociadas con Hiposialia

\begin{tabular}{|l|l|}
\hline Estudio & Hallazgo \\
\hline Hemograma & Anemia \\
\hline Ferritina, acido fólico, vitamina B12 & Anemia ferropriva, megaloblástica \\
\hline Glicemia, PTGO, HbA1c & Diabetes (microangiopatía, neuropatía, xerostomía) \\
\hline Función tiroidea & Hipotiroidismo, hipertiroidismo \\
\hline Anti Ro, Anti La, factor reumatoide, ANA & Sindrome de Sjögren, enfermedades reumatologicas \\
\hline Biopsia de la punta de la lengua & $\begin{array}{l}\text { Alteraciones en las fibras nerviosas epiteliales y subpa- } \\
\text { pilares }\end{array}$ \\
\hline Test de Parche & Dermatitis de contacto alérgica por materiales dentales \\
\hline Endoscopía digestiva & Reflujo gastroesofagico \\
\hline Estudio salival & Xerostomía \\
\hline Estudio hormonal & Menopausia \\
\hline
\end{tabular}

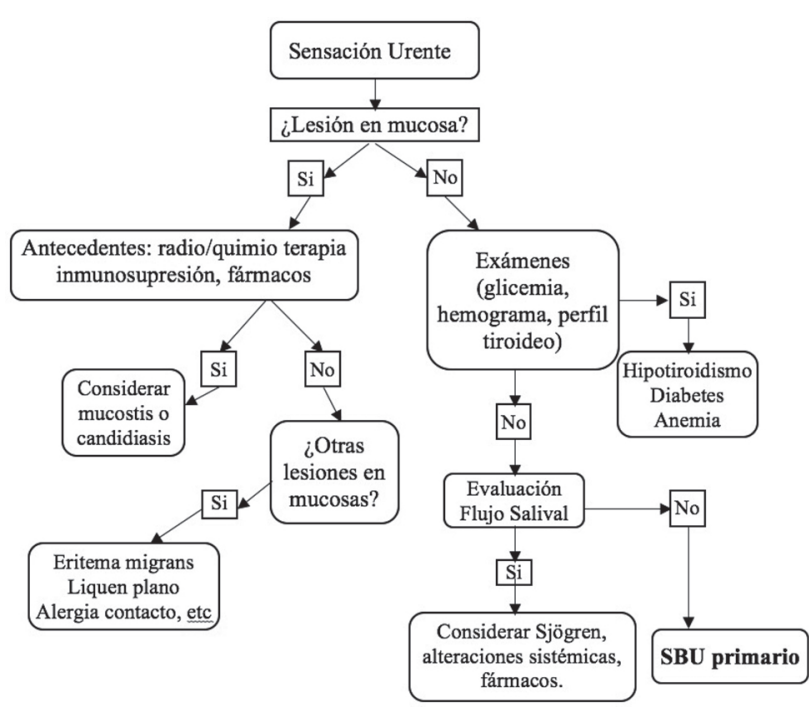

Figura 1

Algoritmo propuesto para el diagnóstico de SBU primario.

Examen físico: Este examen se realizará para descartar lesiones de mucosa oral como eritema, erosiones, lengua depapilada, alteraciones sugerentes de liquen plano, lengua fisurada, lengua geográfica, y otras alteraciones de la apariencia normal de la mucosa. Es importante tener en cuenta que las enfermedades ampollares autoinmunes con frecuencia se inician con molestias en la mucosa oral. La exploración odontológica busca posibles problemas dentales, desajustes de prótesis, galvanismo y alteraciones en el volumen del flujo salival. ${ }^{16}$

\section{EXÁMENES DE LABORATORIO (TABLA 2)}

1. Hemograma: en pacientes con SBU se ha observado hasta en un $13 \%$ la presencia de anemia, debido a que la anemia ferropriva y megaloblástica (por déficit de vitamina B12), se asocian a glositis y atrofia epitelial predisponiendo a la irritación y el dolor.

2. Estudio de diabetes: el examen de glicemia en ayunas, la prueba de tolerancia oral a la glucosa (PTGO) y la HbAlc pueden ser necesarias para descartar neuropatía o microangiopatía diabética. También, pacientes diabéticos mal controlados, presentan un aumento en la diuresis, llevando a la xerostomía y al SBU secundario.

3. Función tiroidea: se ha visto que los síntomas de SBU pueden estar en relación a un hipotiroidismo o también a un hipertiroidismo, por lo que es prudente realizar un estudio de la función tiroidea considerando niveles de TSH, T4 libre y anticuerpos antitiroideos. ${ }^{17}$

4. Otros exámenes sanguíneos: anti-SS-A/ antiSS-Ro, anti-SS-B/anti-SS-La, factor reumatoide y anticuerpos antinucleares deben ser solicitados según la clínica, para evaluar la presencia de síndrome de Sjögren y otras alteraciones reumatológicas. El estudio de la ferritina, ácido fólico y Vitamina B12 también debiesen ser considerados como parte del estudio en caso de sospecharse anemia. 
Tabla 2

Estudio en SBU y posibles hallazgos

\begin{tabular}{llll}
\hline Anticolinérgicos & Atropina & Belladona & Oxibutinina \\
\hline Antidepresivos y antipsicóticos & Citalopram & Venlafaxina & Haloperidol \\
& Fluoxetina & Amitriptilina & Mirtazapina \\
& Paroxetina & Sertralina & Olanzapina \\
\hline Diuréticos & Hidroclorotiazida & Furosemida & Triamtireno \\
\hline Antihipertensivos & Captopril & Enalapril & Metildopa \\
\hline Sedantes y ansiolíticos & Clonidina & & \\
\hline Relajantes musculares & Alprazolam & Diazepam & \\
\hline Analgésicos & Ciclobenzaprina & & \\
\hline Antihistamínicos & Codeina & Ibuprofeno & Piroxicam \\
\hline
\end{tabular}

La biopsia de la punta de la lengua: puede ayudar al diagnóstico evidenciando alteraciones en las fibras nerviosas epiteliales y subpapilares, mediante la inmunohistoquímica y la microscopía confocal, aunque no está indicada en el estudio inicial del SBU.

Test de Parche: se ha observado que algunos pacientes desarrollan el SBU secundario a alergia de contacto a materiales dentales, sobre todo en las presentaciones intermitentes, en las cuales, al eliminar el alérgeno, se elimina la sintomatología. Por lo cual es indispensable el estudio de una respuesta de hipersensibilidad tardía a algún tipo de material dental, siendo los más frecuentes compuestos de la amalgama o de acrílicos utilizados en el tratamiento restaurador de dientes. La indicación de test de parche cutáneo con lectura a las 96 horas es lo que habitualmente se utiliza para la detección de respuestas de hipersensibilidad tardía, sin embargo, reacciones retardadas a metales pudiesen ocurrir hasta los 14 días, por lo que algunos centros incluyen la medición a los 7 y 14 días. ${ }^{18}$

Endoscopía digestiva: El SBU está asociado ocasionalmente al reflujo gastroesofágico, específicamente con la sintomatología de la orofaringe y base de la lengua. Anecdóticamente, se ha reportado alivio de síntomas del SBU con omeprazol dos veces al día, en pacientes con reflujo gastroesofágico (RGE) demostrado por endoscopia. ${ }^{19} \mathrm{Si}$ bien el uso de los inhibidores de la bomba de protones en pacientes con SBU y RGE ha aumentado, puede que no sea necesariamente beneficioso; Becker et al. no observaron conexión causal entre reflujo y sensación de ardor intraoral en una serie de 11 pacientes estudiados con ambas patologías. ${ }^{20}$

Estudio salival: Una de las quejas más frecuentemente asociadas al SBU es la xerostomía, debido a esto, las alteraciones en el flujo salival como causa de SBU secundario deben ser descartadas mediante pruebas de sialometría no estimulada. ${ }^{21}$

Estudio hormonal: en un estudio sobre mujeres con menopausia y molestias orales, Volpe et al encontraron que, con la suplementación de estrógenos, mejoraron los síntomas objetivos y subjetivos en 12 de 22 pacientes tratadas con estriol, y 7 de 10 pacientes tratadas con estrógenos conjugados asociados a noretisterona, por lo que las mujeres con receptores de estrógenos en la mucosa oral, pudieran disminuir la sintomatología con terapia de reemplazo hormonal, no pudiendo garantizarse que sea un tratamiento efectivo. 


\section{Tratamiento}

Aún no se establece un manejo completo y efectivo en el SBU, se ha comprobado en algunos estudios la mejoría con ciertas modalidades de tratamiento, pero no existe un protocolo unificado. Se ha observado que la desaparición espontánea de los síntomas ocurre en muy pocos casos, de 5 a 20\%, y en promedio, sólo el $40 \%$ de los pacientes se benefician de los medicamentos para el dolor neuropático..$^{22,23}$

En caso de ser comprobable una afección local o sistémica, el tratamiento debe ir enfocado es esto, sin embargo, no existiendo una alteración comprobable, ya sea mediante la inspección oral o por medio de exámenes, debiese manejarse como un SBU primario. ${ }^{24}$

Además, habiéndose descartado la causa secundaria, también se debe considerar la prevención de posibles factores irritativos y traumáticos sobre la mucosa oral. Se deben pulir bordes filosos de dientes o prótesis que produzcan irritación en mucosa, incluso considerar algún protector dental que evite la acción irritativa de los movimientos parafuncionales sobre la lengua. Junto con esto, se podría asociar un gel a base de aloe vera, por sus efectos antiinflamatorios y lubricantes tópicos. Siempre tranquilizar al paciente explicando la naturaleza de las molestias.

En casos en los cuales la xerostomía pueda explicar los síntomas, el manejo de esta es primordial. Para esto existen diversas alternativas: saliva artificial, ciclosporina tópica, sialólogos (como el ácido málico y el ácido ascórbico) y medicamentos parasimpaticomiméticos, como la pilocarpina, que demostró eficacia clínica y seguridad en un estudio reciente realizado en Chile, en dosis de $5 \mathrm{mg}$ vía oral, 3 veces al día. ${ }^{25}$

En el paciente con SBU primerio, de ser posible, se ha sugerido intentar discriminar entre una neuropatía periférica o central, mediante la anestesia regional bilateral del nervio lingual. Si a pesar de este procedimiento las molestias mantienen, podríamos atribuir la causa a una alteración de origen central. ${ }^{26}$ En el caso de sospechar un SBU primario de afectación periférica, el uso de clonazepam tópico sería la primera elección. Un meta-análisis reciente concluyó que el clonazepam fue eficaz en la remisión de los síntomas en pacientes con SBU. ${ }^{27}$ Un comprimido de clonazepam de $1 \mathrm{mg}$ tres veces al día, disuelto en la boca por tres minutos y luego escupido, alivió significativamente la intensidad del dolor en comparación con el placebo. ${ }^{26} \mathrm{El}$ uso de clonazepam $0,5 \mathrm{mg}$ sistémico mostró una leve mejoría frente al placebo, lo que sin embargo, no fue estadísticamente significativo. ${ }^{28}$ La combinación de clonazepam tópico y sistémico, habría mostrado un mayor rendimiento, sin embargo el estudio retrospectivo que se realizó este hallazgo requiere confirmación. ${ }^{29}$ Tal vez este mayor efecto este determinado por un SBU con afectación principalmente a nivel central.

El uso de ácido alfa lipoico (AAL) mostró efectos positivos en algunos estudios, pero han sido refutados por otros. Debido a lo heterogéneo de los resultados no se puede apoyar sustancialmente su eficacia en el tratamiento del SBU primario, sin embargo pudiese considerarse, especialmente asociado a gabapentina, donde

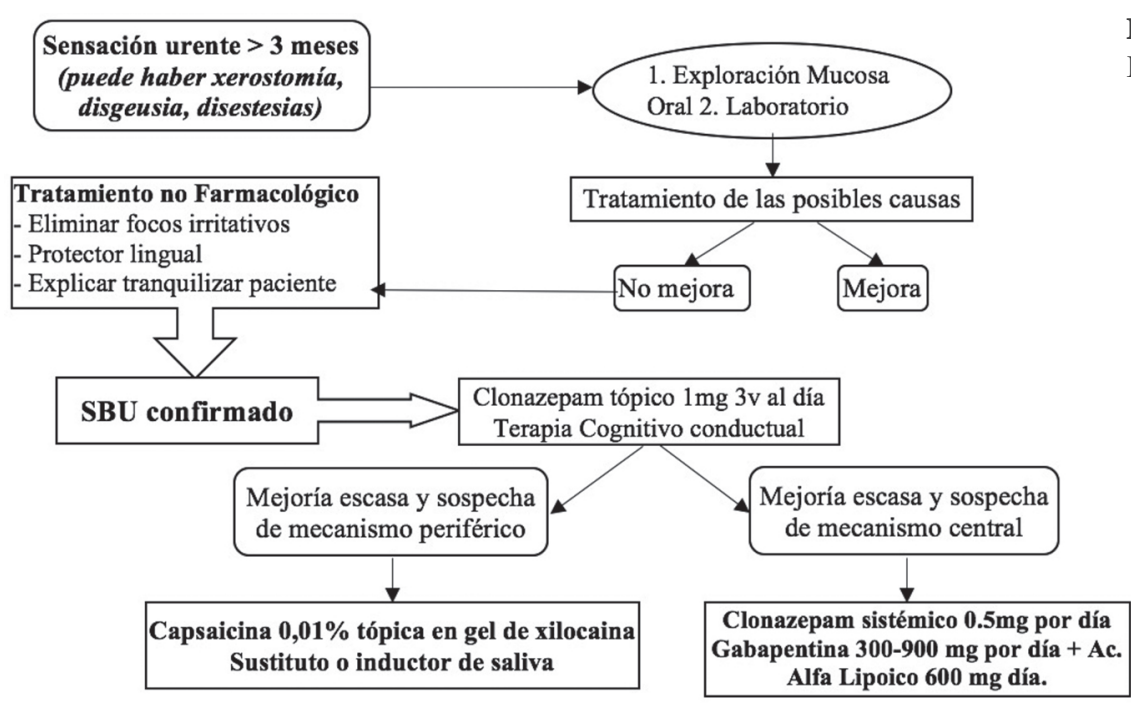

Figura 2

Esquema terapéutico SBU propuesto. 
su efecto podría ser significativamente mayor. Aunque se ha encontrado poco o ningún efecto, la gabapentina y otros anticonvulsivantes se utilizan de manera rutinaria, pudiendo ser una opción en los pacientes con SBU primario. ${ }^{30}$

Además de lo descrito anteriormente, se han propuesto muchas drogas según opinión de expertos y reporte de casos, haciendo eco de los tratamientos para otras neuropatías. Entre ellos se señalan la amitriptilina, con un resultado apenas superior a la mejoría espontánea. El tramadol, que ha sido frecuentemente usado, aunque hay poca evidencia en la literatura que apoye su uso. El uso de tranilcipromina asociada a ansiolíticos e hipnóticos, si bien constituyen una terapia antigua, ha reportado beneficios, siendo aún utilizada en nuestro medio como alternativa para el manejo del SBU. ${ }^{31}$

Por último, varios tratamientos tópicos, como protectores linguales o capsaicina $(0.01 \%)$ con xilocaina $(2 \%)$ formulado en orobase, dos veces al día, puede ser efectivo, especialmente cuando se sospecha un mecanismo periférico como origen del SBU (figura 2).

\section{Conclusión}

El SBU, por sus características etiopatogénicas y clínicas, muchas veces, constituye un desafío, debido a que la causa rehúye los diferentes estudios, y los tratamientos indicados no dan solución, aumentando la frustración en el paciente y con ello su sintomatología, deteriorando su calidad de vida a veces de forma severa. En los últimos años, y gracias el mejor entendimiento de este síndrome, podemos realizar un abordaje que nos permite un mejor manejo, enfocándonos en el estudio y tratamiento de las causas secundarias, o realizando un tratamiento sintomático del SBU como un fenómeno primario. Todo esto, luego de una cuidadosa exploración mediante la anamnesis, el examen físico, los exámenes de laboratorio y las pruebas complementarias. Si la clínica o los hallazgos obtenidos lo ameritan, debe hacerse una derivación oportuna a la especialidad respectiva, para realizar un manejo multidisciplinario. Finalmente, recalcar que no debemos olvidar la esfera psiquiátrica, que puede estar presente y pasar desapercibida incluso para el paciente siendo el trabajo interdisciplinario entre médico, odontólogo y psiquiatra o psicólogo es indispensable.

\section{REFERENCIAS}

1. Merskey H, Bogduk N. Classification of Chronic Pain: Descriptions of Chronic Pain Syndromes and Definitions of Pain Terms. Seattle: IASP Press; 1994:74-75.

2. International Headache Society Classification Committee. The international classification of headache disorders. Cephalalgia 2004; 24(Suppl 1): 1-136.

3. Klasser GD, Pinto A, Czyscon JM, Cramer CK, Epstein J. Defining and diagnosing burning mouth syndrome. J Am Dent Assoc 2013; 144(10): 1135-42.

4. Maltsman-Tseikhin A1, Moricca P, Niv D. Burning mouth syndrome: Will better understanding yield better management? Pain practice 2007; 7(2): 151-62.

5. Suzuki N, Mashu S, Toyoda M, Nishibori M. Oral Burning Sensation: Prevalence and Gender Differences in a Japanese Population. Pain Practice. Pain Pract 2010 Jul-Aug;10(4):306-11.

6. Grushka M., Clinical features of burning mouth syndrome. Oral Surg Oral Med Oral Pathol 1987 Jan; 63(1): 30-6.

7. Aravindhan R, Vidyalakshmi S, Kumar MS, Satheesh C, Balasubramanium AM, Prasad VS. Burning mouth syndrome: A review on its diagnostic and therapeutic approach. J Pharm Bioallied Sci 2014 Jul; 6 (Suppl 1): S21-S25.

8. Jääskeläinen SK, Forssell H, Tenovuo O. Abnormalities of the blink reflex in burning mouth syndrome. Pain 1997;73 (3): 455-60.

9. Lauria G, Majorana A, Borgna M, Lombardi R, Penza P, Padovani A, Sapelli P. Trigeminal small-fiber sensory neuropathy causes burning mouth syndrome. Pain 2005; 115(3): 332-337.

10. Granot M, Nagler RM. Association between regional idiopathic neuropathy and salivary involvement as the possible mechanism for oral sensory complaints. J Pain 2005;6 (9): 581-7.

11. Grushka M., Epstein JB, Gorsky M. Burning mouth syndrome and other oral sensory disorders: a unifying hypothesis. Pain Res Manag 2003;8 (3): 133-5.

12. Jääskeläinen SK, Rinne JO, Forssell $\mathrm{H}$, Tenovuo $\mathrm{O}$, Kaasinen V, Sonninen P, Bergman J. Role of the dopaminergic system in chronic pain -- a fluorodopa-PET study. Pain 2001;90 (3): 257-60.

13. Sreebny LM, Schwartz SS. A reference guide to drugs and dry mouth--2nd edition. Gerodontology 1997; 14(1): 33-47.

14. Brailo V1, Vuéiaeeviae-Boras V, Alajbeg IZ, Alajbeg I, Lukenda J, Aeurkoviae M. Oral burning symptoms and burning mouth syndrome-significance of different variables in 150 patients. Med Oral Patol Oral Cir Bucal 2006; 11(3): E252-5.

15. J. Romaní, D. Chesa. Psicodermatología en atención primaria. Piel 2005; 20(6): 282-289.

16. Redondo G, Brufau M, Corbalán R et al. Síndrome de la boca urente. Actas Dermosifiliogr 2008; 99(6): 431-40.

17. Femiano F1, Gombos F, Esposito V, Nunziata M, Scully C. Burning mouth syndrome (BMS): evaluation of thyroid and taste. Med Oral Patol Oral Cir Bucal. 2006; 11(1): E22-5.

18. Rojas-Alcayaga G, Carrasco-Labra A, Danús P, Guzmán MA, Morales-Bozo I, Urzúa B, Ortega-Pinto A. Determination of susceptibility to sensitization to dental materials in atopic and nonatopic patients. Med Oral Patol Oral Cir Bucal. 2012; 17(2): e320-4. 
19. Garcia-Bravatti M. Re: Burning mouth syndrome. Am J Gastroenterol 1996; 91(6): 1281-1282.

20. Becker S, Schmidt C, Berghaus A, Tschiesner U, Olzowy B, Reichel O. Does laryngopharyngeal reflux cause intraoral burning sensations? A preliminary study. Eur Arch Otorhinolaryngol 2011; 268(9): 1375-81

21. Nagler RM, Hershkovich O. Sialochemical and gustatory analysis in patients with oral sensory complaints. J Pain 2004; 5(1): 56-63.

22. Sardella A, Lodi G, Demarosi F, et al. Causative or precipitating aspects of burning mouth syndrome: a casecontrol study. J Oral Pathol Med 2006; 35(8): 466-71.

23. Finnerup NB, Attal N, Haroutounian S, et al. Pharma- cotherapy for neuropathic pain in adults: A systematic review and meta-analysis. Lancet Neurol 2015; 14(2): 162-173.

24. Cifuentes M, Del Barrio-Díaz P, Vera-Kellet C. Pilocarpine and artificial saliva for the treatment of xerostomia and xerophthalmia in Sjögren syndrome: a double-blind randomized controlled trial. Br J Dermatol. 2018; 179(5): 1056-1061.

25. Jaaskelainen SK, Woda A. Burning mouth síndrome. Cephalalgia 2017; 0(0) 1-21

26. Grémeau-Richard C, Dubray C, Aublet-Cuvelier B, Ughetto S, Woda A. Effect of lingual nerve block on burning mouth syndrome (stomatodynia): a randomized crossover trial. Pain 2010;1 49(1): 2732.

27. Cui Y, Xu H, Chen FM, Liu JL, Jiang L, Zhou Y, Chen QM. Efficacy evaluation of clonazepam for symptom remission in burning mouth syndrome: A meta-analysis. Oral Dis 2016; 22(6): 503-11.

28. Heckmann SM1, Kirchner E, Grushka M, Wichmann MG, Hummel T. A double-blind study on clonazepam in patients with burning mouth syndrome. Laryngoscope 2012; 122(4): 813-6.

29. Amos K, Yeoh SC and Farah CS. Combined topical and systemic clonazepam therapy for the management of burning mouth syndrome: A retrospective pilot study. J Orofac Pain 2011; 25(2): 125-30.

30. Zakrzewska J and Buchanan JA. Burning mouth syndrome. BMJ Clin Evid 2016 Jan 7; 2016. pii: 1301.

31. Grinspan D, Fernández Blanco G, Allevato MA, Stengel FM. Burning mouth syndrome. Int J Dermatol. 1995; 34(7): 483-7. 